SECTION 31. Economic research, finance, innovation, risk management.

Anatoly Aleksandrovich Naumov Docent, Candidate of Technical Sciences, Center of Applied Mathematical Research, Novosibirsk, Russia a_a_naumov@mail.ru

Anastasia Anatolievna Naumova Marketing Director, MBA, Marie Forleo Business School, NYC, Manchester, UK anastasia.naumova@yahoo.com

\title{
ABOUT INCORRECTNESS OF ONE OPTIMIZATION OF CAPITAL STRUCTURE MODEL
}

Abstract: The paper presents the results of one model capital structure optimization study. The disadvantages of this model are shown. Recommendations for improving of the model are presented.

Key words: Capital structure, borrowing, optimization, modeling, financial flows, detailing flows method.

Citation: Naumov AA, Naumova AA (2014) ABOUT INCORRECTNESS OF ONE OPTIMIZATION OF CAPITAL STRUCTURE MODEL. ISJ Theoretical \& Applied Science 9 (17): 170-173. doi: http://dx.doi.org/10.15863/TAS.2014.09.17.29

УДК 336.77: 330.42

\section{О НЕКОРРЕКТНОСТИ ОДНОЙ МОДЕЛИ ОПТИМИЗАЦИИ СТРУКТУРЫ КАПИТАЛА}

Аннотация: В работе приведены результаты исследования модели оптимизации структуры капитала. Показаны недостатки этой модели. Приведень рекомендации по улучшению модели.

Ключевые слова: Структура капитала, заимствования, оптимизация, моделирование, финансовые потоки, метод детализации потоков.

Введение. Взяться за написание настоящей работы нас вынудили исследования модели оптимизации структуры капитала, опубликованные в работах [1] - [3]. Мы всегда считали, что прикладные научные работы вообще (а работы экономико-математического плана - в особенности) должны продвигать (разъяснять, популяризировать) новые интересные идеи для их эффективного практического использования. Они должны в достаточно четком и, по возможности, в простом и понятном для практиков виде демонстрировать притягательные прикладные свойства идей, обращать внимание практиков на подходы (модели, алгоритмы), которые позволят решать их задачи более рационально и эффективно. К сожалению, с работами, в которых излагаются приемы решения экономических задач с использованием математических методов, часто происходит совсем обратное. После ознакомления с такими работами у практиков возникает по поводу решения их задач еще больше вопросов, чем их было до такого знакомства. Думается, что работы [1], [2] обладают именно таким «запутывающим» практиков свойством. В общем, попытаемся объяснить нашу точку зрения аргументировано. 
Задача оптимизации структуры капитала представляется нам очень интересной как с научной, так и с прикладной точек зрения. Она возникает каждый раз, когда решается вопрос: как наилучшим образом объединить в бизнесе финансовые потоки от нескольких источников финансирования? Например, какой объем средств и на каких условиях заимствовать, чтобы в сочетании с собственными средствами это позволило бы получить наибольший эффект для бизнеса? Особенность этой задачи состоит в том, что она должна решаться в рамках более широкой задачи планирования развития бизнеса вообще, она является задачей динамической, в ней присутствуют элементы неопределенности, а значит, и рисков.

Некорректности в математических моделях. Рентабельность собственного капитала. Рассмотрим предлагаемые подходы и модели для решения этой задачи и отметим основные причины, по которым использование этих моделей на практике, к сожалению, не представляется возможным. Начнем с модели для рентабельности собственного капитала [3]:

$$
R_{\mathrm{CK}}(C K, 3 K)=\left[\frac{(1+q / 100)(B-C-K P)+S_{\text {пр }}}{C K+3 K}+\frac{(1+q / 100)(B-C-K P)+S_{\text {пр }}}{C K+3 K}-r\right] \cdot \frac{C K}{3 K} \cdot(1-N) \text {. }
$$

Здесь использованы обозначения (см. [3]): $R_{\mathrm{CK}}(C K, 3 K)$ - рентабельность собственного капитала; $C K$ - величина собственного капитала; $3 K$ - величина заемного капитала; $B$ выручка; $C$ - себестоимость; $K P$ - коммерческие расходы; $S_{\text {пр }}=Д_{\text {пр }}-P_{\text {пр }}$, где $Д_{\text {пр }}-$ прочие доходы; $P_{\text {пр }}$ - прочие расходы; $r$-стоимость заемного капитала $3 K ;(1+q / 100)$ темп роста объемов продаж; $N$ - ставка налога на прибыль.

По поводу этой модели для рентабельности $C K$ следует заметить, что, во-первых, в ней допущена опечатка и отношение $C K / 3 K$ должно стоять множителем внутри скобок следующим образом:

$$
R_{\mathrm{CK}}(C K, 3 K)=\left[\frac{(1+q / 100)(B-C-K P)+S_{\text {пр }}}{C K+3 K}+\left[\frac{(1+q / 100)(B-C-K P)+S_{\text {пр }}}{C K+3 K}-r\right] \cdot \frac{C K}{3 K}\right] \cdot(1-N) \text {, }
$$

и, во-вторых, последнее выражение для $R_{\mathrm{CK}}(C K, 3 K)$ с помощью несложных преобразований существенно упрощается до вида:

$$
R_{\text {СК }}(C K, 3 K)=\frac{\left((1+q / 100)(B-C-K P)+S_{\text {пр }}-r \cdot 3 K\right) \cdot(1-N)}{C K} .
$$

Здесь (в отличие от первоначального вида) смысл критерия $R_{\mathrm{CK}}(C K, 3 K)$ становится понятен: рентабельность собственного капитала $R_{C K}$ находится как отношение средств оставшихся после расчета за заемный капитал и уплаты налогов $\left((1+q / 100)(B-C-K P)+S_{\text {пр }}-r \cdot 3 K\right) \cdot(1-N)$, к объему собственных средств $(C K)$ (см. [4] - [6]). Возникает несколько вопросов по поводу модели: 1) зачем нужно было авторам так существенно усложнять вид выражения для $R_{C K}(C K, 3 \mathrm{~K})$ ?; 2) почему рентабельность оценивается только относительно собственного капитала без учета заемного? Ведь прибыль будет получена от использования обоих видов капиталовложений в бизнес (CK и $3 K)$. 
Ставка по заемным средствам и погашение по заимствованиям. В выражении для рентабельности $R_{\mathrm{CK}}(C K, 3 K)$, использована ставка по заемным средствам $r$, а произведение $r \cdot 3 K$ означает, какую сумму придется оплатить за использование заемного капитала в объеме $3 K$, и поэтому это произведение вычитается из полученной прибыли. Так, в примере (см. [3]) использовано значение $r=0.1$ (т.е. в следующий временной период придется вернуть заемщику $10 \%$ от заемного капитала). Возникает вопрос: $r \cdot 3 K$ - это проценты за использование $3 K$, а почему не учитывается возврат самих заимствований (в размере $3 K)$ ? Конечно, возвращать за заимствование придется сумму в размере $(1+r) \cdot 3 K$

. Это значение и нужно было использовать в формулах для рентабельности, времени оборота капитала и пр. Заметим, что обычно на практике погашение заемных средств и процентов по ним происходит не в один такт времени, а растягивается во времени на несколько временных тактов. В этом случае предлагаемые в [3] расчетные формулы придется существенно изменить, и использовать, например, схемы аналогичные приведенным в работе [7].

Понижение размерности задачи. Вполне понятно, что размерность математической модели играет существенную роль как на этапе анализа этой модели, так и на этапе ее использования при решении реальных задач. Можно утверждать, что модель для рентабельности $R_{\text {СK }}(C K, 3 K)$ и построенная на ее основе оптимизационная задача (см. [3]) имеют размерность равную не двум, а единице. Это так, поскольку общий объем капиталовложений на прогнозируемый период известен и он равен сумме $K_{\text {пр.акт }}=C K+3 K$. Тогда неизвестной является только одна из переменных $(C K$ или $3 K)$ и другая может быть найдена из последнего равенства. Итак, модель и оптимизационная задача в [3] являются одномерными, что существенно упрощает их анализ и решение.

Еще одно уменьшение размерности можно осуществить за счет сокращения количества критериев в оптимизационной задаче. Один из критериев этой задачи - это $R_{\mathrm{CK}}(C K, 3 K)$, а другой - время оборота капитала выглядит следующим образом:

$$
T_{\text {об }}(C K, 3 K)=\frac{C K+3 K}{\left((1+q / 100)(B-C-K P)+S_{\text {пр }}-r \cdot 3 K\right) \cdot(1-N)} \cdot
$$

Легко заметить, что он представляет собой величину обратную для рентабельности всего объема капитала, т.е. для $R_{\mathrm{CK}+3 к}(C K, 3 K)$. С учетом того, что именно эту рентабельность и следует оценивать, один из критериев может быть исключен из оптимизационной задачи. Таким образом, оптимизационная задача в [3] фактически является однокритериальной и зависит от одной переменной.

Собственный капитал. В работе [3] не рассматривается вопрос об использовании остатков собственного капитала в текущем периоде. Это очень важный момент, поскольку эти остатки необходимо использовать полностью или частично в прогнозном периоде, и представляется неэффективным, если часть этого капитала будет «заморожена», например, на расчетном счете банка. Фактически, относительно $C K$ должна решаться задача оптимального его перераспределения между разными бизнесами (проектами). В любом случае, будет известен объем собственных средств, используемых в данном бизнесе. Эта задача похожа на задачу оптимизации перераспределения финансовых средств между различными проектами, рассмотренную в [7], [8].

Заемный капитал. Практические нюансы работы с заемными средствами таковы, что расчет за них, как правило, осуществляется за несколько временных тактов. Причем, заимствования необходимо производить с учетом наличия на прогнозируемый период 
объемов собственного капитала. Учитывать это обстоятельство можно, например, так, как это предложено в работах [7], [8],[10].

Как должна решаться (и решается!) на практике задача оптимизации структуры капитала. Распишем схематично работу алгоритма.

1. Необходимо построить прогноз для объемов продаж на предстоящий период времени, т.е., фактически, необходимо оценить значение $q$;

2. Зная прогнозируемое значение объемов продаж, необходимо пересчитать общий объем требуемого капитала на предстоящий период $K_{\text {пр.акт }}=C K+3 K$;

3. Зная стратегию развития бизнеса, следует оценить размер собственного капитала, который будет доступен для вложения в бизнес на предстоящий период (т.е. оценить $C K$ );

4. Определив необходимый объем заимствования ( $\left.3 K=K_{\text {пр.акт }}-C K\right)$ ), найти (подобрать) наилучший в соответствии с некоторыми критериями эффективности (доход, доходность, рентабельность и пр.) вариант заимствования (сроки, ставки, объемы заимствований из разных источников и т.д.); в этом случае необходимо решить задачу перебора конечного числа вариантов заимствования капитала в размере $3 K$.

\section{References:}

1. Kirillov UV, Nazimko EN (2011) Mnogokriterial'naya model' optimizacii struktury kapitala. Ekonomicheskiy analiz: teoriya i praktika, No. 32, pp. 57-63.

2. Kirillov UV, Nazimko EN (2013) Reshenie mnogokriterial'noy zadachi optimizacii struktury kapitala. Ekonomicheskiy analiz: teoriya i praktika, No. 28, pp. 55-63.

3. Kirillov UV, Nazimko EN (2014) Analiz resheniya mnogokriterial'noy zadachi optimizacii struktury kapitala. Finansovaya analitika: problemy i resheniya. No. 25, pp. 34-41.

4. Naumov AA (2013) K analiticheskim resheniyam nekotoryh ekonomiko-matematicheskih zadach. Materials of the ISPC «Results \&Perspectives», 30.09.2013, Florence, Italy.

Theoretical \& Applied Science 9 (5), pp. 93-97. doi: http://dx.doi.org/10.15863/TAS.2013.09.5.11

5. Naumov AA (2013) Optimizaciya struktury zaimstvovaniy i vlozheniy dohodov investicionnogo proekta. Materials of the ISPC «Advances in techniques\&technologies», 30.10.2013, Milan, Italy. Theoretical \& Applied Science 10(6), pp. 133-136. doi: http://dx.doi.org/10.15863/TAS.2013.10.6.20

6. Naumov AA (2013) K voprosu ob uproschenii dvuh zadach optimizacii investiciy. Finansovaya analitika: problemy i resheniya, No.46 (184), pp. 26-30.

7. Naumov AA (2013) Ispol'zovanie metoda detalizacii finansovyh potokov $v$ zadachah ocenivaniya ieffektivnosti proektov. Finansovaya analitika: problemy i resheniya, No. 48 (186), pp. 35-41.

8. Naumov AA (2014) Ocenivanie ieffektivnosti integrirovannyh proektov. Finansovaya analitika: problemy i resheniya, No. 8 (194), pp. 36-43.

9. Sharp U, Aleksandr G, Beyli Dzh (2001) Investicii. Moscow: INFRA-M, pp. 1028.

10. Naumov AA (2013) Metody analiza i sinteza investicionnyh proektov. Effektivnost', riski, upravlenie. LAP LAMBERT Academic Publishing, pp. 356.

11. Spisok trudov (2014) Available: https://sites.google.com/site/anatolynaumov2011/home/spisok-trudov-list-of-papers Accessed: 2014 Sep 25. 